\title{
Estratégias para incremento de carbono e retorno econômico em áreas de sequeiro com sistemas de integração Lavoura-Pecuária-Floresta no semiárido
}

Strategies for increasing carbon and economic return in rainfed areas with crop-livestock-forest integration systems in the semiarid region

Estrategias para incrementar el carbono y la rentabilidad económica en zonas de secano con sistemas de integración cultivo-ganadería-bosque en la región semiárida

Arlen Nicson Lopes Pena

ORCID: https://orcid.org/0000-0001-7817-7170

Universidade Federal de Minas Gerais, Brasil

E-mail: nicsonlopes1547@gmail.com

Cássia Michele Cabral

ORCID: https://orcid.org/0000-0002-4730-4509 Universidade Federal dos Vales do Jequitinhonha e Mucuri, Brasil

E-mail: mtchells@gmail.com

Leidivan Almeida Frazão

ORCID: https://orcid.org/0000-0001-6848-9007

Universidade Federal de Minas Gerais, Brasil

E-mail: leidivan.frazao@gmail.com

\begin{abstract}
Resumo
Os sistemas integrados de produção agropecuária podem promover melhoria da qualidade física, química e biológica do solo. A obtenção de efeitos sinérgicos entre os diferentes componentes de produção permite a sustentabilidade dos sistemas agropastoril, silvipastoril, agroflorestal e agrossilvipastoril. Aumentos na estocagem de carbono podem ser obtidos a partir da implantação de sistemas integrados de produção, além de maiores retornos econômicos. A diversificação da produção possibilita a redução de riscos frente às oscilações de preços de mercado. Contudo, estudos envolvendo a estocagem de carbono e, principalmente, a viabilidade econômica de sistemas integrados cultivados em região semiárida são limitados. Neste contexto, objetivou-se apresentar resultados de estocagem de carbono e retorno econômico obtidos com a adoção de sistemas integrados de produção no semiárido, visando apresentar resultados que possam subsidiar a implementação de projetos futuros, permitindo a obtenção de ganhos nos estoques de carbono e maior viabilidade econômica em condições de baixa disponibilidade hídrica.
\end{abstract}

Palavras-chave: Matéria orgânica do solo; Viabilidade econômica; Sistema agrossilvipastoril.

\section{Abstract}

Integrated agricultural production systems can improve the physical, chemical and biological quality of the soil. Obtaining synergistic effects between the different production components allows the sustainability of the agropastoral, silvopastoral, agroforestry and agrosilvopastoral systems. Increases in carbon storage can be obtained 
with the adoption of integrated production systems, in addition to greater economic returns. The diversification of production makes it possible to reduce risks in the face of fluctuations in market prices. However, studies involving carbon storage and, mainly, the economic viability of integrated systems in the semiarid region are limited. In this context, the objective was to present results of carbon storage and economic return obtained with the adoption of integrated production systems in the semiarid region, aiming to present results that can subsidize the implementation of future projects, allowing to obtain gains in carbon stocks and greater economic viability in conditions of low water availability.

Keywords: Soil organic matter; Economic viability; Agrosilvopastoral system.

\section{Resumen}

Los sistemas integrados de producción agrícola pueden mejorar la calidad física, química y biológica del suelo. La obtención de efectos sinérgicos entre los distintos componentes productivos permite la sostenibilidad de los sistemas agropastoril, silvopastoril, agroforestal y agrosilvopastoril. Se pueden obtener incrementos en el almacenamiento de carbono con la adopción de sistemas de producción integrados, además de mayores retornos económicos. La diversificación de la producción permite reducir los riesgos ante las fluctuaciones de los precios de mercado. Sin embargo, los estudios que involucran el almacenamiento de carbono y, principalmente, la viabilidad económica de los sistemas integrados en la región semiárida son limitados. En este contexto, el objetivo fue presentar resultados de almacenamiento de carbono y retorno económico obtenidos con la adopción de sistemas de producción integrados en la región semiárida, con el objetivo de presentar resultados que puedan subsidiar la implementación de proyectos futuros, permitiendo obtener ganancias en stocks de carbono y mayor viabilidad económica en condiciones de escasa disponibilidad de agua.

Palabras clave: Materia orgánica del suelo; Viabilidad económica; Sistema agrosilvopastoril.

\section{Introdução}

A produção agrícola, pecuária e florestal pode promover reduções significativas na qualidade do solo (Freitas et al., 2020; Serpa et al., 2020). A busca pela sustentabilidade da produção agropecuária e florestal tem se intensificado frente a necessidade da preservação dos recursos naturais e de melhorias em questões sociais e econômicas. Neste contexto, os sistemas integrados têm sido colocados como alternativas sustentáveis de produção. Menezes et al. (2012) destacam a importância da adoção de sistemas de uso da terra baseados em espécies perenes (componente florestal) e com maior biodiversidade.

De acordo com Cordeiro et al. (2015), os sistemas integrados associam os componentes agrícola, pecuário e florestal em uma mesma área, seja em consórcio, sucessão ou rotação. Estes sistemas, segundo Gontijo Neto et al. (2014), compreendem a integração Lavoura-Pecuária (ILP), Pecuária-Floresta (IPF), Lavoura-Floresta (ILF) e Lavoura-PecuáriaFloresta (ILPF), podendo, também, ser denominados por agropastoril, silvipastoril, agroflorestal e agrossilvipastoril, respectivamente. Diferentes estudos mostraram a obtenção de aumento da fertilidade, da qualidade física e da atividade biológica a partir da adoção dos sistemas integrados de produção em diferentes condições edafoclimáticas (Abreu et al., 2020; Almeida et al., 2021; Assis et al., 2015, Stieven et al., 2014; Bonini et al., 2016).

Adicionalmente às melhorias obtidas na qualidade física, química e biológica do solo, Barbosa et al. (2015) apontaram os sistemas de integração Lavoura-Pecuária e integração Lavoura-Pecuária-Floresta como alternativas economicamente viáveis e atrativas, possibilitando uma produção agrícola sustentável, necessária frente ao crescimento populacional e à necessidade de preservação do meio ambiente. De acordo com os autores, esses sistemas podem amortizar custos, por exemplo, de reforma de pastagens, propiciando resultados econômicos positivos em médio e longo prazo.

Estudos envolvendo a estocagem de carbono e, principalmente, a viabilidade econômica de sistemas integrados na região semiárida são limitados. Alterações na matéria orgânica do solo decorrentes da adoção de práticas alternativas de produção foram estudadas em região semiárida por Assis et al. (2011). Os autores reportaram a obtenção de maiores estoques de carbono orgânico do solo em sistemas agrossilvipastoris e silvipastoris. Contudo, verificaram-se a necessidade de realização de estudos envolvendo questões agronômicas, econômicas e sociais associadas aos benefícios ambientais verificados com a prática da agrossilvicultura para, assim, classificá-la como estratégia de produção sustentável para o semiárido.

Segundo Ferraza et al. (2016), a maioria dos estudos de viabilidade econômica dos sistemas integrados foram 
realizados em condições climáticas favoráveis ao desenvolvimento da agropecuária ou em regiões com cadeias produtivas estabelecidas, com maior valor de mercado. Neste contexto, objetivou-se apresentar resultados de estocagem de carbono e retorno econômico obtidos com a adoção de sistemas integrados de produção no semiárido, visando apresentar resultados que possam subsidiar a implementação de projetos futuros, permitindo a obtenção de ganhos nos estoques de carbono e maior viabilidade econômica em condições de baixa disponibilidade hídrica.

\section{Metodologia}

Foi realizada uma pesquisa bibliográfica (pesquisa quantitativa) extensa e detalhada sobre o tema abordado (Pereira et al., 2018). Neste sentido, as estratégias para incremento de carbono e para a obtenção de retorno econômico em áreas de sequeiro com sistemas de integração Lavoura-Pecuária-Floresta no semiárido foram estudadas a partir de publicações (entre os anos de 2004 e 2021) nacionais e internacionais, pesquisadas em diferentes bases de dados. Foram utilizados trabalhos indexados em: Academia.edu, Agricola, Agricultural Economics Database, AGRIS, Agrobase, base de dados da Pesquisa Agropecuária Brasileira (EMBRAPA), CAB, Crossref, DOAJ, EBSCO, Google Scholar Metrics, Latindex, MEDLINE/PubMed, Qualis/CAPES, Redalyc, REDIB, ResearchGate, SciELO, Scopus e Web of Science.

As principais variáveis utilizadas para avaliar o incremento de carbono foram o estoque de carbono orgânico do solo e a emissão de $\mathrm{CO}_{2}$. Já a avaliação do retorno econômico, deu-se a partir da utilização de indicadores de eficiência econômica comumente utilizados em análises financeiras, dentre os quais foram empregados o custo da produção (CP), o lucro bruto (LB), o valor presente líquido (VPL), valor periódico equivalente (VPE), a taxa de retorno do investimento (TIR) e a relação benefício custo (B/C).

\section{Resultados e Discussão}

\subsection{Incremento de carbono em áreas de sequeiro com sistemas de iLPF no semiárido}

Estoques de carbono sob diferentes sistemas de gestão foram avaliados por Barros et al. (2014) no Sertão Paraibano. Foram estudados os sistemas de pastagem, agricultura e permanente, vegetação esparsa e nativa. Os autores verificaram que a substituição da vegetação nativa por sistemas agrícolas reduziu significativamente os estoques de carbono orgânico do solo. Além disso, foi possível observar aumento das emissões de $\mathrm{CO}_{2}$ nos sistemas agrícola e pecuária comparativamente à vegetação nativa (Caatinga). Dessa forma, constata-se a necessidade de adoção de sistemas de caráter conservacionista em condições semiáridas, visando a sustentabilidade deste ecossistema.

Aguiar et al. (2014) estudaram os sistemas agrossilvipastoril, silvipastoril, cultivo tradicional, áreas de pousio e uma área de Caatinga preservada, objetivando verificar os estoques de carbono presentes nas plantas, serapilheira e solo. Os diferentes sistemas situaram-se na região semiárida brasileira (Estado do Ceará). No sistema agrossilvipastoril, foram cultivados milho e sorgo nas entrelinhas de Leucena (Leucaena leucocephala) e Gliricídia (Gliricidia sepium), na presença de espécies arbóreas nativas. Neste sistema, fêmeas ovinas e caprinas realizaram pastejo durante o período seco. A ausência de cultivo de milho e sorgo no sistema silvipastoril contribuiu com o aumento do estoque de carbono orgânico total do solo comparativamente ao sistema agrossilvipastoril. Os autores relataram que o revolvimento do solo, adotado como prática de manejo das culturas agrícolas, acelera a decomposição dos resíduos orgânicos, reduzindo o estoque de carbono orgânico do solo. Já a ausência de mobilização e a cobertura permanente do solo favorece o acúmulo de carbono. Em relação aos demais sistemas estudados, os sistemas integrados de produção apresentaram reservas de nutrientes e estoques de carbono intermediários entre Caatinga preservada e sistemas tradicionais. De acordo com o estudo, redução de $16 \%$ nos estoques totais de carbono foi verificada no cultivo tradicional, comparativamente ao sistema agrossilvipastoril. 
Aguiar et al. (2014) apontaram os sistemas integrados como alternativa para a mitigação de emissão de gases de efeito estufa, pois apresentaram alto potencial de sequestro de carbono no semiárido brasileiro. Verificaram-se potencial de mitigação de emissão de $\mathrm{CO}_{2}$ em sistema silvipastoril e agrossilvipastoril de 5,0 e 8,8 $\mathrm{t} \mathrm{ha}^{-1}$ ano $^{-1}$, respectivamente. Adicionalmente, o estudo reportou que os sistemas integrados possibilitam a diminuição da necessidade de exploração da vegetação nativa devido à intensificação sustentável da produção, contribuindo indiretamente com o sequestro de carbono, pois a criação de novas áreas de produção, envolvendo a mudança do uso da terra, promove elevada emissão de gases de efeito estufa. O potencial de sequestro de carbono foi associado a maior produção de biomassa. Moraes et al, (2017) evidenciaram a necessidade de preservação dos remanescentes de vegetação de Caatinga como meio de contribuir com a redução de emissões de gases de efeito estufa e, consequentemente, com a mitigação do aquecimento global. Marin et al. (2006) correlacionaram o acúmulo de carbono orgânico do solo com o aporte de resíduos orgânicos sobre o solo, sendo o teor de matéria orgânica regulado pela quantidade e pela taxa de decomposição do material orgânico.

Estoque de carbono em diferentes sistemas agrícolas foram estudados por Sacramento et al. (2013) em região semiárida do Brasil. Foram avaliaram os sistemas agrossilvipastoril, Silvipastoril, cultivo tradicional e vegetação nativa (Caatinga) na profundidade de $0-60 \mathrm{~cm}$. O sistema agrossilvipastoril foi composto por 200 árvores nativas ha ${ }^{-1}$ e por linhas de Leucena espaçadas por $3 \mathrm{~m}$ de distância. Entre as linhas de Leucena, foi cultivado milho na estação chuvosa, enquanto na estação seca houve pastejo com ovelhas. Já o sistema silvipastoril foi composto por 260 árvores nativas ha ${ }^{-1}$. No sistema tradicional realizou-se o cultivo de milho em estações chuvosas. O sistema agrossilvipastoril proporcionou maior armazenamento de carbono orgânico em relação aos sistemas silvipastoril e convencional após 13 anos de produção. A agricultura convencional promoveu perdas de $58,87 \mathrm{Mg} \mathrm{ha}^{-1}$ no estoque de carbono orgânico do solo. $\mathrm{O}$ estudo apontou o sistema agrossilvipastoril como estratégia de conservação dos estoques de carbono do solo em região semiárida, possibilitando uma produção agrícola, pecuária e florestal sustentável.

Os baixos aportes de biomassa vegetal, os reduzidos índices pluviométricos, as práticas de queimada e a mobilização do solo estão entre as principais práticas que contribuem com o baixo conteúdo de matéria orgânica do solo geralmente encontrado em regiões semiáridas. Neste contexto, sistemas integrados de produção devem ser utilizados visando uma maior cobertura do solo, a manutenção da umidade e, consequentemente, a redução de processos erosivos, promovendo melhoria da qualidade física, química e biológica do solo (Assis et al., 2011). Pinheiro e Nair (2018) recomendaram a manutenção de pelo menos $40 \%$ do componente forrageiro após o pastejo como forma de proteção do solo, reduzindo, assim, perda de qualidade em condições semiáridas.

\subsection{Retorno econômico em áreas de sequeiro com sistemas de iLPF no semiárido}

Os riscos da atividade agrícola e da atividade pecuária podem ser reduzidos a partir da diversificação da produção, ou seja, os retornos econômicos podem ser favorecidos a partir da integração de diferentes componentes de produção, tendo como exemplo a integração Lavoura-Pecuária. Mesmo em curto prazo, a integração pode promover resultados econômicos mais atrativos comparativamente a sistemas únicos de produção, como monocultivo de lavoura ou criação de gado, propiciando redução da relação risco/retorno esperados (Lazzarotto et al., 2009).

Cobucci et al. (2007) atribuem a viabilidade econômica em sistemas integrados de produção ao melhor aproveitamento dos recursos de produção disponíveis na propriedade rural. Dentre os recursos citados pelos autores, destacamse a área de produção (terra) e maquinários existentes; o sinergismo entre os diferentes sistemas de produção, como o aproveitamento dos resíduos agrícolas como fonte de alimento para animais, o aporte de nitrogênio proveniente da fixação biológica resultante da associação simbiótica entre leguminosas e rizóbios, que favorece as forrageiras cultivadas na mesma área. Outro sinergismo ocorre por meio da reciclagem de nutrientes pelas forrageiras, cultivadas posteriormente às lavouras 
que são manejadas com utilização de fertilizantes químicos. Além disso, tem-se a diversificação de receitas decorrente do cultivo de diferentes componentes de produção. Verifica-se também a diminuição de custos de produção devido a menor necessidade de insumos, como defensivos e fertilizantes. A maior conservação do solo e da água em sistemas integrados permite, ainda, redução de custos de manejo devido a menor necessidade de práticas reparadoras/corretivas.

Drumond et al. (2004) investigaram diferentes sistemas agroflorestais no semiárido brasileiro, constatando os consórcios de leucena x sorgo, Eucalyptus camaldulensis x capim urocloa, algarobeira x agave, algarobeira x capim buffel, gliricídia x palma e umbuzeiro x palma como alternativas tecnicamente viáveis. Contudo, os autores recomendaram a realização de análises econômicas para avaliação da sustentabilidade de produção destes sistemas nas condições citadas. De acordo com o estudo, a viabilidade econômica pode ser obtida com a venda dos grãos, lenha ou forragem. Com isso, caso seja verificado ganho superior aos custos de implantação e manutenção, o sistema poderá ser considerado economicamente viável.

Santana Neto et al. (2005), em estudo que avaliou leguminosas adapatadas como alternativa alimentar para ovinos no semiárido, recomendaram o cultivo de Gliricídia (Gliricidia sepium), Leucena (Leucena leucocephala), Algaroba (Prosopis juliflora) e Sabiá (Mimosa caesalpiniifolia) visando aumento de produtividade sob condições de restrição hídrica e, consequentemente, proporcionando maior lucratividade na criação de ovinos. As leguminosas citadas apresentam, segundo os autores, alternativa proteica de baixo custo, pois são espécies tolerantes à baixa disponibilidade hídrica e possuem alto valor forrageiro.

Sistemas de integração Lavoura-Pecuária foram estudados no semiárido mineiro por Ferrazza et al. (2016), objetivando avaliar a viabilidade bioeconômica do monocultivo de sorgo e do consórcio de sorgo com diferentes forrageiras, em diferentes espaçamentos entre linhas de plantio. Os autores estimaram os custos de produção em função dos valores praticados no mercado regional no momento do estudo. As receitas foram determinadas com base na venda de grãos e com o aluguel de pastagens. Foi obtido o fluxo de caixa e determinada a viabilidade econômica a partir da diferença entre receitas e custos, calculando-se, ainda, indicadores de eficiência econômica comumente utilizados em análises financeiras. O indicador valor presente líquido (VPL) foi positivo para os consórcios de sorgo com Chenchus ciliares, sorgo com Brachiaria brizantha e sorgo com Brachiaria ruziziensis, ambos no espaçamento de $40 \mathrm{~cm}$. No espaçamento de $80 \mathrm{~cm}$, verificaram-se VPL positivo nos consórcios de sorgo com Andropogon gayanus e sorgo com Brachiaria ruziziensis, indicando viabilidade econômica, já que apresentaram VPL positivo.

O monocultivo de sorgo mostrou-se inviável economicamente, pois obteve VPL negativo em ambos os espaçamentos $(40$ e $80 \mathrm{~cm}$ ). As taxas de retorno do investimento (TIR), calculadas nos consórcios citados, foram superiores à taxa de juros utilizada no estudo (6\% a.a.), reforçando a viabilidade econômica desses sistemas integrados, fato não verificado para o TIR obtido nos cultivos solteiros de sorgo. A relação benefício custo dos tratamentos em consórcio citados foram superiores a 1, indicando retorno econômico positivo, sendo os maiores valores verificados nos consórcios de Sorgo com Brachiaria brizantha em $40 \mathrm{~cm}(\mathrm{~B} / \mathrm{C}=3,19)$ e sorgo com Brachiaria ruziziensis $\mathrm{em} 80 \mathrm{~cm}(\mathrm{~B} / \mathrm{C}=3,33)$.

França et al. (2011) analisaram a viabilidade econômica da exploração de ovinos e caprinos no Estado do Ceará Brasil em sistema agrossilvipastoril com 50 ha e 280 animais. Por meio do fluxo de caixa do sistema, foram apresentados indicadores econômicos para demonstração da possibilidade de retorno econômico da atividade estudada. Os autores constataram viabilidade econômica do projeto, possibilitando sobrevivência digna de produtores rurais em condições semiáridas associada a sustentabilidade ambiental da Caatinga. Verificaram-se VPL positivo, com TIR de 52,03\% e relação $\mathrm{B} / \mathrm{C}$ de 1,59, indicando que a cada 1,00 R \$ investido houve retorno de 1,59 R\$.

Mendes (2016) avaliaram um sistema de ILPF localizado no município de Francisco Sá - MG, Brasil. No sistema, foram cultivados sorgo, eucalipto e capim braquiária. O sorgo foi cultivado no primeiro (juntamente com o plantio de eucalipto) e segundo ano de condução do sistema. No segundo ano, o sorgo foi consorciado com braquiária e, após 2,5 anos de 
implantação do sistema de ILPF, houve pastejo de gado. Para avaliação do sistema integrado localizado na região semiárida mineira, foi levantado o fluxo de caixa do projeto e foram calculados os indicadores econômicos VPL, TIR, B/C, Payback e valor periódico equivalente (VPE). Verificou-se VPL (-352,02 R \$ ha- $\left.{ }^{-1}\right)$ e VPE (-55,02 R\$ ha ${ }^{-1}$ ano $\left.^{-1}\right)$ negativos e TIR (7\% a.a.) inferior a taxa de juros $(8,75 \%$ a.a.), além de $\mathrm{B} / \mathrm{C}(0,96)$ menor que 1 . Apesar do período necessário para recuperação do investimento realizado para implantação do sistema (payback $=7,26$ anos) ter ficado dentro do tempo planejado (10 anos de condução do projeto), o sistema foi considerado economicamente inviável. Verificou-se influência positiva do componente arbóreo com a previsão de venda de madeira aos 6 anos de cultivo e dos animais a cada ano. Porém, o sorgo influenciou negativamente a viabilidade econômica do sistema, devido aos altos custos para implantação desta cultura. De acordo com o estudo, a integração dos diferentes componentes permite compensar o baixo preço de determinado produto com a venda de outro produto, que esteja em alta naquele momento, consistindo em uma importante estratégia frente a instabilidade de preços de mercado.

Silva et al. (2015) compararam os benefícios econômicos de um sistema agroflorestal em relação à agricultura convencional em Nova Olinda, no Ceará - Brasil. O sistema agroflorestal foi composto por espécies florestais, frutíferas e medicinais, sendo a produção escalonada ao longo do ano, enquanto no sistema convencional houve cultivo de milho, feijão e fava. Para a comparação econômica, foram determinados os índices valor bruto da produção (VBP), custo da produção (CP), lucro bruto (LB) e relação Benefício/Custo (B/C). Os índices estudados demonstraram maior viabilidade econômica com a adoção do sistema agroflorestal, comparativamente à agricultura convencional. A relação B/C no sistema integrado foi 2,18 vezes superior ao cultivo de lavoura, demonstrando que o sistema agroflorestal estudado possibilitou melhores condições para enfrentamento das adversidades climáticas presentes em condições semiáridas.

De acordo com Ferraza et al. (2016), a baixa ou a inviabilidade econômica observada em sistemas de produção pode ocorrer em função dos altos preços de insumos (fertilizantes, defensivos agrícolas, sementes, entre outros), dos diferentes níveis de degradação do solo (baixa qualidade física, química e biológica) e em função dos baixos índices pluviométricos verificados em regiões semiáridas, que comprometem o rendimento das culturas. Com isso, nota-se a necessidade de realização de planejamento dos sistemas integrados de produção com foco nas condições locais, buscando alternativas que viabilizem a obtenção de ganhos ambientais e econômicos. A utilização de espécies animais e vegetais adaptadas à baixa disponibilidade hídrica associadas a manejos que propiciem a obtenção de efeitos sinérgicos entre os diferentes componentes do sistema devem ser priorizados, visando a redução de custos e a obtenção de ganhos de produção. Conforme Mendes (2016), os sistemas de ILPF podem resultar ou não em retorno econômico positivo, o qual exige adequado planejamento, investimento e infraestrutura existente na propriedade rural, assim como acesso a conhecimento técnico, condições climáticas adequadas às espécies trabalhadas e perfil do produtor.

\section{Considerações Finais}

Os sistemas de ILPF podem promover maior estocagem de carbono quando comparados aos sistemas exclusivos de produção agrícola em condições semiáridas, assim como podem favorecer maior retorno econômico. Contudo, exige-se maior planejamento para que sejam obtidos resultados positivos. Deve-se, portanto, priorizar a utilização de espécies vegetais e animais tolerantes às condições de restrição hídrica, o emprego de estratégias que maximizem o aproveitamento dos efeitos sinérgicos que podem ser obtidos com a integração de diferentes componentes de produção, a verificação de condições mercadológicas favoráveis à produção (acessibilidade e preço de insumos) e comercialização dos produtos gerados (tipos de grãos, carne, mercado acessível para aquisição de madeira) e a existência de aptidão e conhecimento do produtor em relação às atividades desenvolvidas. Tais estratégias podem influenciar diretamente a sustentabilidade ambiental e econômica dos 
sistemas integrados e devem, deste modo, ser consideradas no planejamento e execução dos sistemas integrados de produção em regiões semiáridas, dadas as limitações desses locais.

Trabalhos futuros devem ser realizados visando o estudo de espécies vegetais e animais tolerantes às condições de restrição hídrica em sistemas integrados com o objetivo de se maximizar a estocagem de carbono e o retorno econômico em regiões semiáridas, frente à necessidade de ampliação e aprofundamento das informações existentes acerca do tema em questão.

\section{Referências}

Abreu, L. H. G., Freitas, I. C., Santana, P. H. L., Barbosa, D. L. A., Santos, L. D. T., Santos, M. V., Sanglard, D. A., \& Frazao, L. A. (2020). Variation in soil carbon, nitrogen and microbial attributes within a silvopastoral system in the Brazilian Cerrado. Agroforestry Systems, 94(6), $2343-2353$.

Aguiar, M. I., Fialho, J. S., Campanha, M. M., \& Oliveira, T. S. (2014). Carbon sequestration and nutrient reserves under different land use systems. Revista Árvore, 38(1), 81-93.

Almeida, L. L. S., Frazão, L. A., Lessa, T. A. M., Fernandes, L. A., Veloso, A. L. C., Lana, A. M. Q., Souza, I. A., Pegoraro, R. F., \& Ferreira, E.A. (2021). Soil carbon and nitrogen stocks and the quality of soil organic matter under silvopastoral systems in the Brazilian Cerrado. Soil and Tillage Research, 205, 104785 .

Assis, C. D., Xavier, F. D. S., Maia, S. M. F., Nogueira, R. S., Alencar, G. D., \& Oliveira, T. D. (2011). Soil organic matter changes in agroforestry and organic farming in the semi-arid region of northeastern Brazil. Dynamic Soil, Dynamic Plant, 5(1), 36-44.

Assis, P. C., Stone, L. F., Medeiros, J. C., Madari, B. E., Oliveira, J. D. M., \& Wruck, F. J. (2015). Atributos físicos do solo em sistemas de integração lavoura-pecuária-floresta. Revista Brasileira de Engenharia Agrícola e Ambiental, 19(4), 309-316.

Barbosa, F. A., Costa, P. M., Andrade, V. J., Maia Filho, G. H. B., Maciel, I. C. F., \& Lopes, S. Q. (2015). Avaliação econômica e produtiva dos sistemas integrados de lavoura-pecuária-floresta. Caderno de Ciências Agrárias, 7(1), 151-165.

Barros, J. D. S., Chaves, L. H. G., \& Pereira, W. E. (2015). Carbon and nitrogen stocks under different management systems in the Paraiban "Sertão". African Journal of Agricultural Research, 10(3), 130-136.

Bonini, C. D. S. B., Lupatini, G. C., Andrighetto, C., Mateus, G. P., Heinrichs, R., Aranha, A. S., Santana, E. A. R., \& Meirelles, G. C. (2016). Produção de forragem e atributos químicos e físicos do solo em sistemas integrados de produção agropecuária. Pesquisa agropecuária brasileira, 51(9), 1695-1698.

Cobucci, T., Wruck, F. J., Kluthcouski, J., Muniz, L. C., Martha Júnior, G. B., Carnevalli, R. A., Teixeira, S. R., Machado, A. A., \& Teixeira Neto, M. L. (2007). Opções de integração lavoura-pecuária e alguns de seus aspectos econômicos. Informe Agropecuário, 28(240), 64-79.

Cordeiro, L. A. M., Vilela, L., Marchão, R. L., Kluthcouski, J., \& Martha Júnior, G. B. M. (2015). Integração lavoura-pecuária e integração lavoura-pecuáriafloresta: estratégias para intensificação sustentável do uso do solo. Cadernos de Ciência \& Tecnologia, 32(1/2), 15-53.

Drumond, M. A., Morgado, L. B., Ribaski, J., Albuquerque, S. G., \& Carvalho Filho, O. M. (2004). Contribuição da Embrapa Semi-Árido para o desenvolvimento dos sistemas agroflorestais no semi-árido brasileiro. Agrossilvicultura, 1(2), 145-153.

Ferrazza, R. A., Lopes, M. A., \& Albuquerque, C. J. B. (2016). Avaliação bioeconômica do consórcio de sorgo com diferentes espécies forrageiras para sistema de integração lavoura-pecuária em Nova Porteirinha, MG. Boletim de Indústria Animal, 73(2), 94-102.

França, F. M. C., Holanda Júnior, E. V., \& Sousa Neto, J. M. (2011). Análise da viabilidade financeira e econômica do modelo de exploração de ovinos e caprinos no Ceará por meio do Sistema Agrossilvipastoril. Revista Econômica do Nordeste, 42(2), 287-308.

Freitas, L., Oliveira, I. A., Souza, S. C., Reis, I. M. S., \& Campos, M. C. C. (2020). Análise visual da qualidade estrutural de solos com diferentes sistemas de uso e manejo no Marajó, Município de Breves, Estado do Pará, Brasil. Research, Society and Development, 9(8), e470985504-e470985504.

Gontijo Neto, M. M., Viana, M. C. M., Alvarenga, R. C., Santos, E. A., Simão, E. D. P., \& Campanha, M. M. (2014). Sistemas de integração lavoura-pecuáriafloresta em Minas Gerais. Boletim de Indústria Animal, 71(2), 183-191.

Lazzarotto, J. J., Santos, M. L., Lima, J. E., \& Moraes, A. (2009). Volatilidade dos retornos econômicos associados à integração lavoura-pecuária no Estado do Paraná. Revista de Economia e Agronegócio, 7(2), 259-284.

Marin, A. M. P., Menezes, R. S. C., Silva, E. D., \& Sampaio, E. V. D. S. B. (2006). Efeito da Gliricidia sepium sobre nutrientes do solo, microclima e produtividade do milho em sistema agroflorestal no Agreste Paraibano. Revista Brasileira de Ciência do Solo, 30(3), 555-564.

Mendes, R. T. Avaliação econômica e de risco de investimento em três sistemas agroflorestais no Norte de Minas e no Alto Jequitinhonha. (2016). Dissertação (Mestrado - Programa de Pós-Graduação em Ciência Florestal) - Universidade Federal dos Vales do Jequitinhonha e Mucuri.

Menezes, R. S. C., Sampaio, E. V. S. B., Giongo, V., \& Pérez-Marin, A. M. (2012). Biogeochemical cycling in terrestrial ecosystems of the Caatinga Biome. Brazilian Journal of Biology, 72(3), 643-653.

Morais, Y. C. B., Araújo, B., Socorro, M., Moura, M. S. B., Galvíncio, J. D., \& Miranda, R. Q. (2017). Análise do Sequestro de Carbono em áreas de Caatinga do Semiárido Pernambucano. Revista Brasileira de Meteorologia, 32(4), 585-599. 
Research, Society and Development, v. 10, n. 7, e48710716769, 2021

(CC BY 4.0) | ISSN 2525-3409 | DOI: http://dx.doi.org/10.33448/rsd-v10i7.16769

Pereira, A. S., Shitsuka, D. M., Parreira, F. J., \& Shitsuka, R. (2018). Metodologia da pesquisa científica. UFSM, NTE.

Pinheiro, F. M., \& Nair, P. K. R. (2018). Silvopasture in the Caatinga biome of Brazil: A review of its ecology, management, and development opportunities. Forest systems, 27(1), 1-16.

Sacramento, J. A. A. S. D., Araújo, A. C. D. M., Escobar, M. E. O., Xavier, F. A. D. S., Cavalcante, A. C. R., \& Oliveira, T. S. D. (2013). Soil carbon and nitrogen stocks in traditional agricultural and agroforestry systems in the semiarid region of Brazil. Revista Brasileira de Ciência do Solo, 37(3), 784-795.

Santana Neto, J. A., Oliveira, V. S., \& Valença, R. L. (2015). Leguminosas adaptadas como alternativa alimentar para ovinos no semiárido-revisão. Revista de Ciências Agroveterinárias, 14(2), 191-200.

Serpa, K. M., Monteiro, F. N., Falcão, K. S., Menezes, R. S., Ferreira, R. S., \& Panachuki, E. (2020). Atributos físicos e teor de matéria orgânica em área de Cerrado sob diferentes sistemas de cultivo. Research, Society and Development, 9(3), e131932399-e131932399.

Silva, T. T., Drumond, M. A., Bakke, I. A., \& Silva, S. M. (2015). Performance econômica e ambiental de agricultura agroflorestal e convencional no município de Nova Olinda-CE. Revista Verde de Agroecologia e Desenvolvimento Sustentável, 10(5), 85-91.

Stieven, A. C., Oliveira, D. A., Santos, J. O., Wruck, F. J., \& Campos, D. T. D. S. (2014). Impacts of integrated crop-livestock-forest on microbiological indicators of soil. Revista Brasileira de Ciências Agrárias, 9(1), 53-58. 